\title{
Ultrasound guided aspiration biopsy for pulmonary tuberculosis with unusual radiographic appearances
}

Ang Yuan, Pan-Chyr Yang, Dun-Bing Chang, Chong-Jen Yu, Li-Na Lee, Huey-Don Wu, Sow-Hsong Kuo, Kwen-Tay Luh

\begin{abstract}
Background Pulmonary tuberculosis can produce unusual radiographic appearances and negative results of sputum and bronchoscopic examinations are common. This study assessed the value of ultrasound guided aspiration biopsy in the diagnosis of pulmonary tuberculosis with unusual radiographic appearances.
\end{abstract}

Methods Thirteen patients, ultimately diagnosed as having tuberculosis, underwent a chest ultrasonographic examination between June 1984 and August 1991. All had sputum available for examination and nine were also examined by bronchoscopy. Ten patients who had a negative sputum smear and negative bronchoscopic brushing smears underwent ultrasound guided aspiration or biopsy. Percutaneous aspiration was performed with a 22 gauge needle. If the smear did not reveal acid fast bacilli, a biopsy sample was taken with a 16 gauge Tru-cut needle to obtain a histological diagnosis.

Results The ultrasonographic examination delineated the more complex nature of the lesions better than the chest radiograph. Ultrasound guided aspiration biopsy provided the diagnosis in nine of 10 patients, while the sputum smear and culture provided diagnosis in five of 13, and bronchoscopy in four of nine. In terms of rapid diagnosis, ultrasound guided aspiration biopsy gave the diagnosis in eight of 10 cases. No patient developed a major complication.

Conclusion Ultrasonography can direct the needle to the most suitable part of a lesion to obtain the relevant specimens. The diagnostic yield is high and the procedure is relatively safe. It is especially helpful in patients with negative results of sputum and bronchoscopic examinations.

(Thorax 1993;48:167-170)

The chest radiographic appearances of pulmonary tuberculosis are variable. ${ }^{1}$ Unusual radiological appearances such as a mass, homogeneous consolidation, atelectasis, or lesions occurring in unusual locations may occur in $15-30 \%$ of patients with active pul- monary tuberculosis. ${ }^{12}$ In these circumstances, radiographic findings are of limited value for a presumptive diagnosis, and confirmation of the aetiology will depend upon microbiological examination. ${ }^{3}$ While sputum smears for acid fast bacilli and sputum culture for Mycobacterium tuberculosis are often positive, sputum negative pulmonary tuberculosis is still a commonly encountered problem. ${ }^{45}$ Microbiological examination of bronchoscopic washings, brushings, lavage specimens, and histological examination of biopsy specimens are also sometimes diagnostic in sputum negative pulmonary tuberculosis. $^{5-10}$ Ultrasound guided aspiration biopsy has been reported to be a satisfactory method for obtaining specimens suitable for histological diagnosis in patients with pulmonary, mediastinal, and pleural lesions with various causes. ${ }^{12-17}$

This study retrospectively analysed the chest ultrasonographic findings in 13 cases of pulmonary tuberculosis presenting with unusual radiographic appearances, and compared the diagnostic yield of ultrasound guided aspiration and biopsy with that of sputum examination and bronchoscopic procedures.

\section{Methods}

Thirteen patients with unusual radiographic appearances of active pulmonary tuberculosis, all of whom had undergone chest ultrasonographic examination, were investigated at the National Taiwan University Hospital between June 1984 and August 1991. Nine were men of mean age 53.5 years (range 18-86 years). The criteria for patient selection included chest radiographic appearances which did not suggest pulmonary tuberculosis, such as a mass $(n=3)$, homogeneous consolidation $(n=7)$, and atelectasis $(n=3),{ }^{12}$ and the absence of any bleeding tendency. The diagnosis of pulmonary tuberculosis was made by either: a positive culture for $M$ tuberculosis from sputum, bronchoscopic washing specimen, needle aspirate and cutting biopsy specimen; demonstration of acid fast staining bacilli in the specimen; or histological demonstration of granulomatous inflammation with Langhan's giant cells and caseation.

All patients had sputum examinations when possible, including acid fast stain and culture 
Figure 1 (A)

Posterioanterior chest radiograph of a 41 year old woman showing lobar consolidation of the right lower lobe. (B) Chest ultrasonography through the right posterior eighth intercostal space showing a lobar distributed

hypoechoic consolidation with ill defined margin

(black arrowheads).

Prominent hyperechoic air bronchograms are scattered in the consolidation (white arrowheads).
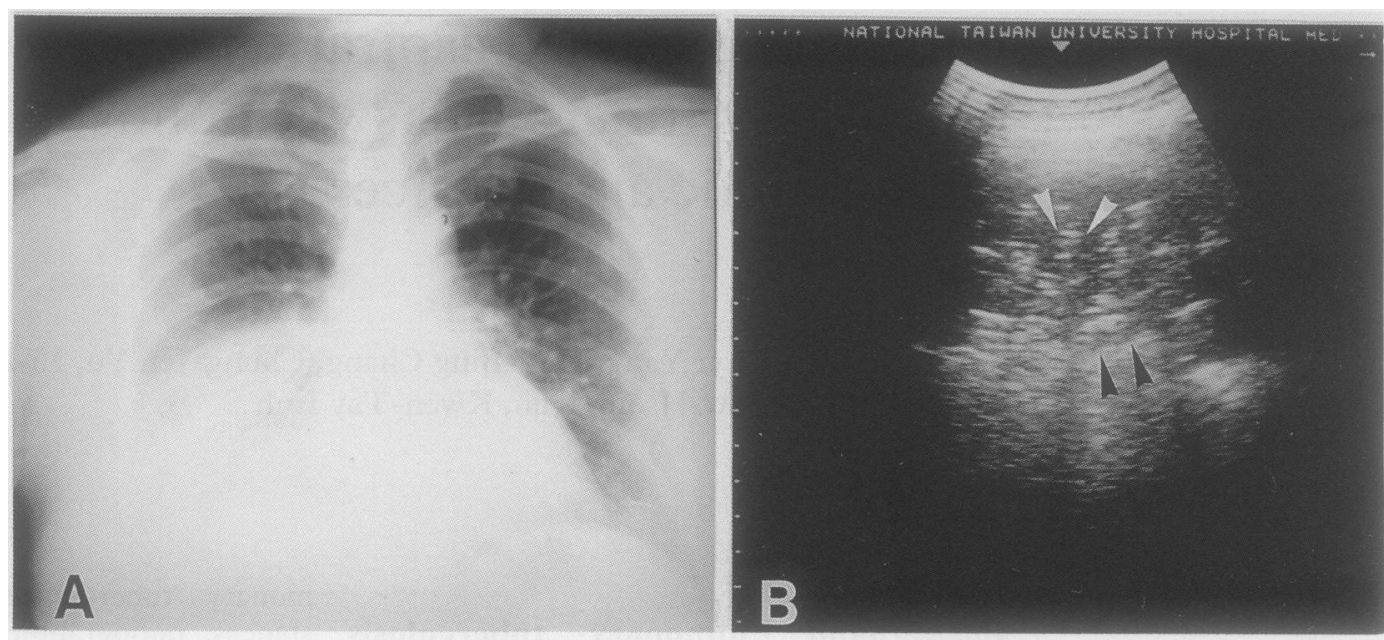

for $M$ tuberculosis from up to three consecutive specimens. In nine patients fibreoptic bronchoscopy was performed, including bronchoscopic brushing, washing, and transbronchial lung biopsy. The specimens obtained from bronchoscopic procedures were sent for cytological examination, acid fast stain, Löwenstein cultures for $M$ tuberculosis and pathological examination. Ten patients who had negative sputum smears and bronchoscopic brushings then underwent ultrasound guided aspiration and/or cutting biopsy. The specimens obtained by aspiration biopsy were examined as above.

Ultrasonographic examination was performed with a high resolution, real time, ultrasound unit (Aloka SSD 280, SSD 630, SSD 650, Aloka, Tokyo; Toshiba 100A, Toshiba, Tokyo) with 3.5 and $5.0 \mathrm{MHz}$ linear and convex transducers. The location of the lesion was assessed from the chest radiograph, and the transducer was placed over this region. After detection of the lesion, both longitudinal and transverse images were obtained to assess the configuration and patterns of the lesions. ${ }^{13}$

Ultrasound guided aspiration was performed with a special puncture probe (Aloka UST-507 BP). as described in detail else- where. ${ }^{13}$ A 22 gauge needle with an outlet sheath and inner stylet was chosen for aspiration. According to Dutt and Stead et al, a cavitating lesion contains a larger population of tubercle bacilli $\left(10^{7}-10^{9}\right)$ than other pulmonary lesions $\left(10^{4}-10^{5}\right) \cdot{ }^{18}$ We therefore chose primarily cavities for aspiration if they were visible by ultrasonography. Alternatively, we aspirated an area away from air bronchograms and vascular structures in consolidative and obstructive pneumonia, and the central porinitial cytological examination and acid fast staining were negative, percutaneous cutting biopsy was performed with a 16 gauge TruCut needle. ${ }^{19}$ The patients were asked to hold their breath for 15-20 seconds during the aspiration and/or biopsy procedure. They were then observed for one hour, and a chest radiograph was taken the next day.

\section{Results}

The ultrasonographic findings in the seven patients with consolidative pneumonia revealed a wedge shaped isoechoic or hypoechoic lesion with prominent air bronchograms and irregular margins (fig 1). Hyperechoic cavitary tion of mass lesions. When the results of the
Figure 2 (A)

Posterioanterior chest radiograph of an 85 year old man showing partial collapse of the right upper lobe.

(B) Ultrasound scan of the lesion of the chest showing a pattern of obstructive pneumonia : a wedge shaped homogeneous isoechoic lesion (arrows) with dilated hypoechoic fluid bronchograms

(arrowheads) extending from the hilum to the periphery of the lung. The specimen obtained by ultrasound guided TruCut needle biopsy revealed caseous necrosis with Langhan's giant cells.
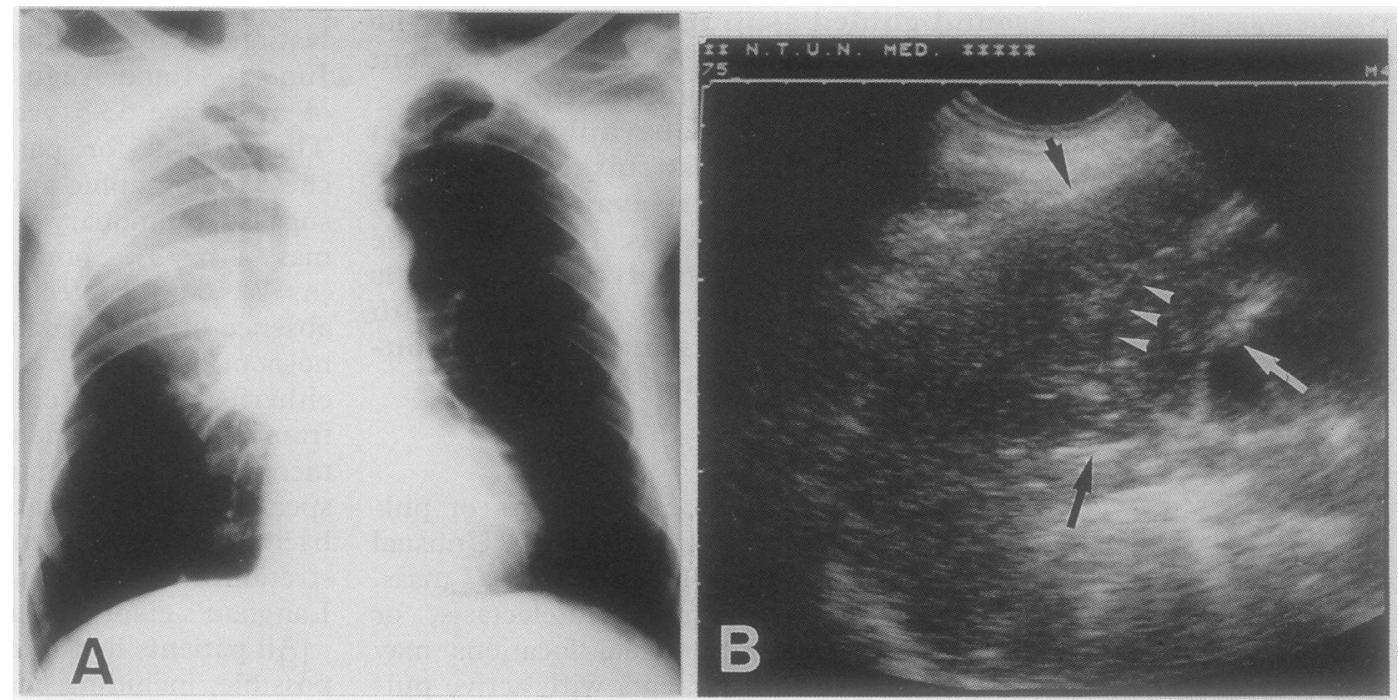
Figure 3 Chest ultrasonograph of a 37 year old man showing the pattern of obstructive pneumonia. A central compression mass $(3.0 \times 3.0 \mathrm{~cm})$ is noted near the hilar area (arrow). Two small areas of liquification (arrowhead) are demonstrated in this compression mass.

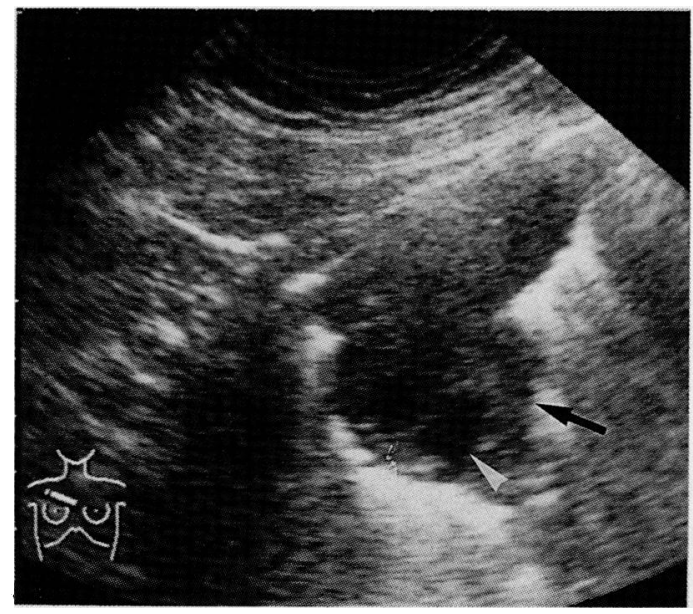

lesions were demonstrated within the consolidation in three cases. In the three patients with obstructive pneumonia, ultrasonography revealed wedge shaped homogeneous lesions, both hyperechoic or isoechioc, with hypoechoic fluid bronchograms arising from the hilum and extending to the periphery of the lung (fig 2). ${ }^{19}$ A centrally located mass was demonstrated in one patient (fig 3), and multiple cavities were noted in another. In the three patients with a pulmonary mass, ultrasonography disclosed a hypoechoic mass lesion with irregular margins (fig 4) ${ }^{13}$ Both large and small cavities were found in these lesions.

Sputum culture for $M$ tuberculosis was positive in five of the 13 patients, but sputum smears for acid fast bacilli was positive in only two. Nine patients underwent bronchoscopy, four patients were diagnosed as having $M$ tuberculosis infection (one by bronchoscopic brush smear and wash culture, one by wash culture alone, one by biopsy histology, one by wash culture and biopsy histology). Ten patients with negative sputum smears and bronchoscopic brushing smears underwent ultrasound guided aspiration and/or biopsy, and in nine a positive diagnosis was made by acid fast staining, culture or histological examination of the specimens. In patients with negative sputum smears, bronchoscopy was diagnostic in one, ultrasound guided needle aspiration in six, and ultrasound guided biopsy in a further two patients. The results showed that ultrasound guided aspiration from a cavitary lesion gave a higher diagnostic yield (4/ 4) than non-cavitating areas of lesions $(2 / 6)$. The diagnostic yields obtained by three different methods in each pattern of the lesions are shown in the table. In cases of consolidative pneumonia, a positive diagnosis was provided by sputum in four of seven patients, by bronchoscopic procedures in four of six patients, and by ultrasound guided aspiration and biopsy in three of four patients. In obstructive pneumonia, only one of three patients was diagnosed by sputum examination, none by bronchoscopic procedures, but all by ultrasound guided aspiration and biopsy. None of the patients with a pulmonary mass was diagnosed by sputum or bronchoscopy examination, but in all three patients the diagnosis was made by ultrasound guided aspiration and biopsy.

Rapid diagnosis was obtained by sputum examination in only two of 13 patients, by bronchoscopic procedure in three of nine patients, and by ultrasound guided aspiration and biopsy in eight of 10 patients.

Only one patient developed mild haemoptysis after ultrasound guided aspiration biopsy of the consolidation area. This subsided spontaneously six hours later. No major complication such as pneumothorax was encountered after aspiration and biopsy.

\section{Discussion}

This study has shown that the ultrasonographic findings of pulmonary tuberculosis with unusual radiographic appearances were even more diverse than the plain radiographs had depicted. In consolidated lesions ultrasonography revealed two patterns: consolidation alone, and consolidation with both large and small cavities not seen on chest radiography. In obstructive lesions ultrasonography

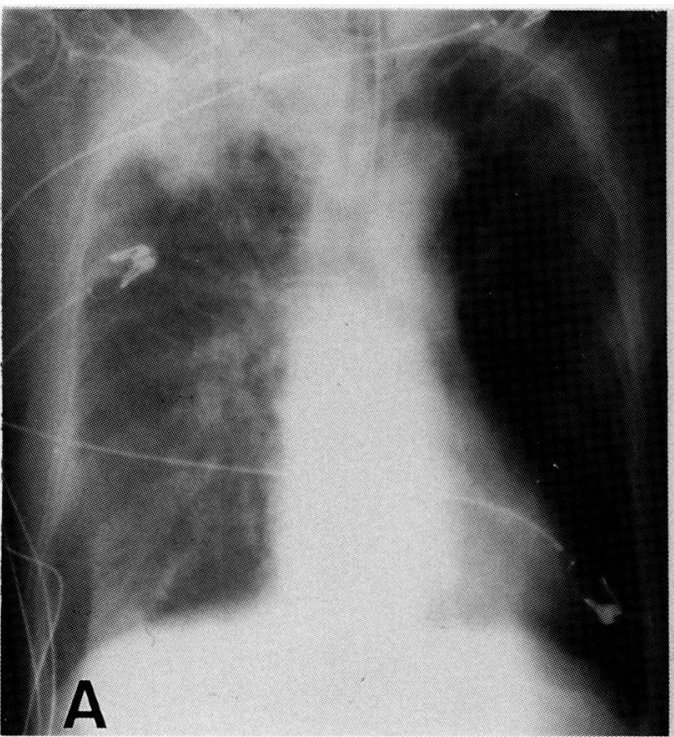

Figure 4 (A)

Posterioanterior chest film of an 86 year old man showing a mass lesion $(5.5 \times 6.0 \mathrm{~cm})$ in the right upper lobe anterior segment. (B) Transverse chest ultrasonograph through the right anterior second intercostal space showing a $4.0 \times 3.0 \mathrm{~cm}$ hypoechoic mass with irregular margins

(arrow). Several small cavities (arrowhead) are noted in this lesion. The acid fast staining of the aspirate obtained by ultrasound guided aspiration revealed acid fast bacilli. 
Diagnostic yields obtained by three methods in three patterns of lesions

\begin{tabular}{llll}
\hline Lesions & Sputum & Bronchoscopy & $\begin{array}{l}\text { Transthoracic } \\
\text { aspiration } \\
\text { and biopsy }\end{array}$ \\
\hline Consolidative pneumonia & $4 / 7$ & $4 / 6$ & $3 / 4$ \\
Obstructive pneumonia & $1 / 3$ & $0 / 2$ & $3 / 3$ \\
Mass & $0 / 3$ & $0 / 1$ & $3 / 3$ \\
Total & $5 / 13$ & $4 / 9$ & $9 / 10$ \\
\hline
\end{tabular}

revealed airway obstruction, and some lesions had multiple cavities. In mass lesions, ultrasonography demonstrated central cavitation. While the ultrasonographic appearance is not specific for pulmonary tuberculosis, ultrasonography can provide more detailed information about the nature of a lesion, and thus indicate the most appropriate area, such as a cavity, for aspiration. Penetration through vascular structures and air or fluid filled bronchi can also be avoided in consolidative and obstructive pneumonia lesions under ultrasound guided aspiration and biopsy.

Our results have shown that ultrasound guided aspiration and biopsy can provide a useful additional technique to sample a lesion directly and to obtain adequate specimens for the diagnosis of tuberculosis. It is especially helpful in those patients with negative sputum and bronchoscopy findings.

For a mass lesion, because of its peripheral location, ultrasound guided aspiration and biopsy are preferable to the other two procedures for diagnosis. ${ }^{13}$ In consolidative pneumonia the three methods had similar yields because of the extensive nature of the lesion. Interestingly, in obstructive pneumonia or atelectasis sputum and bronchoscopy provided a low positive yield. This may be due to the regional lymph node or the granulation tissue causing complete obstruction of the bronchus, making the microorganisms less available for sputum or bronchoscopic sampling.

Transthoracic needle aspiration under fluoroscopic or computed tomography guidance may be an alternative method for diagnosis of pulmonary tuberculosis with unusual radiographic appearances. Under fluoroscopic guidance, however, the fine structures and complex nature of the lesion may not be delineated clearly. Computed tomography is superior to ultrasonography for demonstrating the complex nature of the lesion, but high cost, radiation exposure, and lack of bedside availability make it less convenient for practical use.

Ultrasonography also has its limitations, especially for centrally located lesions without distal pathology. In these lesions ultrasound guided sampling is impossible, as there is no acoustic window through which the ultrasound waves can penetrate.

1 Khan MA, Kovnat DM, Bachus B, Whitcomb ME, Brody JS, Snider GL. Clinical and roentgenographic spectrum of pulmonary tuberculosis in the adults. Am J Med 1977; 62:31-8.

2 Miller WT, MacGregor RR. Tuberculosis: frequency of unusual radiographic findings. AJR 1978;130:867-75.

3 Escreet BC, Cowie RL. Criteria for the diagnosis of pulmonary tuberculosis. $S$ Afr Med J 1983;63:850-4.

$4 \mathrm{Kim} \mathrm{TCH}$, Blackman RS, Heatwole KM, Kim T, Rochester DF. Acid-fast bacilli in sputum smears of patients with pulmonary tuberculosis. Am Rev Respir Dis 1984;129. 264-8.

5 de Gracia J, Curull V, Vidal R, Riba A, Orriols R, Martin N. Diagnostic value of bronchoalveolar lavage in suspected pulmonary tuberculosis. Chest 1988;93:329-32.

6 So SY, Lam WK, Yu DYC. Rapid diagnosis of suspected pulmonary tuberculosis by fiberoptic bronchoscopy. Tubercle 1982;63:195-200.

7 Wallace JM, Deutsch AL, Harrel JH, Moser KM. Bronchoscopy and transbronchial biopsy in evaluation of patients with suspected active tuberculosis. $A m \mathrm{~J} M e d$ 1981;70:1189-94.

8 Willcox PA, Benatar SR, Potgieter PD. Use of the flexible fibreoptic bronchoscope in diagnosis of sputum negative pulmonary tuberculosis. Thorax 1982;37:598-601.

9 Stover DE, Zaman MB, Hajdu SI, Lange MI, Gold J, Armstrong D. Bronchoalveolar lavage in the diagnosis of diffuse pulmonary infiltrates in the immunosuppressed host. Ann Intern Med 1984;101:1-7.

10 Khoo KK, Meadway J. Fibreoptic bronchoscopy in rapid diagnosis of a sputum smear negative pulmonary tuberculosis. Respir Med 1989;83:335-8.

11 Robicheaux G, Moinuddin SM, Lee LH. The role of aspiration biopsy cytology in the diagnosis of pulmonary tuberculosis. Clin Pathol 1985;83:719-22.

12 Matalon TA, Neiman HL, Mintzer RA. Noncardiac ches sonography: the state of the art. Chest 1983;83:675-8.

13 Yang PC, Luh KT, Sheu JC, Kuo SH, Yang SP. Peripheral pulmonary lesions: ultrasonography and ultrasonically guided aspiration biopsy. Radiology 1985;155:451-6.

14 Wernecke K, Vassallo P, Peters PE, von Bassewitz DB. Mediastinal tumors: biopsy under ultrasound guidance. Radiology 1989;172:473-6.

15 Yu CJ, Yang PC, Chang DB, Wu HD, Lee LN, Luh KT. Evaluation of ultrasonically guided biopsies of mediastinal masses. Chest 1991;100:399-405.

16 Chang DB, Yang PC, Luh KT, Kuo SH, Yu CJ. Ultrasound-guided pleural biopsy with Tru-Cut needle. Chest 1991;100:1328-33.

17 Yang PC, Chang DB, Yu CJ, Lee YC, Kuo SH, Luh KT. Ultrasound-guided percutaneous cutting biopsy for the diagnosis of pulmonary consolidation of unknown etiology. Thorax 1992;47:457-60.

18 Dutt AK, Stead WW. Present chemotherapy for tuberculosis. J Infect Dis 1982;146:698-704.

19 Yang PC, Luh KT, Wu HD, Chang DB, Lee LN, Kuo SH. Lung tumors associated with obstructive pneumonitis. Ultrasound studies. Radiology 1990;174:717-20. 